\title{
Application of computational fluid dynamics on cavitation in journal bearings
}

\author{
Marco Riedel $^{1, a}$, Marcus Schmidt ${ }^{2}$, Peter Reinke ${ }^{1}$, Matthias Nobis ${ }^{1}$, and Marcel Redlich ${ }^{2}$ \\ 1 West Saxon University of Applied Science Zwickau, Dr.-Friedrichsring 2A, 08056 Zwickau (Germany) \\ 2 Forschungs- und Transferzentrum e.V., POB 2010 37, 08012 Zwickau (Germany)
}

\begin{abstract}
Journal bearings are applied in internal combustion engines due to their favourable wearing quality and operating characteristics. Under certain operating conditions damage of the journal bearing can occur caused by cavitation. The cavitation reduces the load capacity and leads to material erosion. Experimental investigations of cavitating flows in dimension of real journal bearing are difficult to realize or almost impossible caused by the small gap and transient flow conditions. Therefore numerical simulation is a very helpful engineering tool to research the cavitation behaviour. The CFD-Code OpenFOAM is used to analyse the flow field inside the bearing. The numerical cavitation model based on a bubble dynamic approach and requires necessary initial parameter for the calculation, such as nuclei bubble diameter, the number of nuclei and two empirical constants. The first part of this paper shows the influence of these parameters on the solution. For the adjustment of the parameters an experiment of Jakobsson et.al. [1] was used to validate the numerical flow model. The parameters have been varied according to the method Design of Experiments (DoE). With a defined model equation the parameters determined, to identify the parameter for CFD-calculations in comparison to the experimental values. The second part of the paper presents investigations on different geometrical changes in the bearing geometry. The effect of these geometrical changes on cavitation was compared with experimental results from Wollfarth [2] and Garner et.al. [3].
\end{abstract}

\section{Introduction}

The hydrodynamic journal bearing is an established mechanical element in internal combustion engines. It stands out by the fact of bear high loads, nearly wearless and low noise operating conditions. The simple design and the low priced manufacturing are further advantages. Like in other hydrodynamic applications cavitation is still a problem in journal bearings. Caused by the cavitation, erosion of the surface can occur and that can result in the breakdown of the bearing. To prevent the damage of the journal bearing it is necessary to have knowledge about the cause of cavitation. Only if vapour appears in the flow, cavitation can occur. Therefore investigation of the flow in the bearing with considering of vaporisation is necessary.

For that investigations the computational fluid dynamics (CFD) is used. Cavitation is founded by three dimensional (3D) effects. Therefore the simulations are realised with the 3D-CFD-Code OpenFOAM. This is a excellent tool due to the free availability and the open solver code. The advantage of the numerical simulation is that every detail of the flow can be obtained. Experiments cannot offer this diversity. On the other hand an experiment is necessary to validate the numerical results. One step at the preparation of the simulation is the grid generation. Therefore special

\footnotetext{
a e-mail: marco.riedel@fh-zwickau.de
}

regions has to be considered. A further step in the preparation is the adaptation of the solver on the flow conditions in a journal bearing geometry. Therefore the experiment of Jakobsson et.al. [1] is choosen to simulate different parameter variations according to the method Design of Experiments (DoE). The configuration with the best results is used for a further simulation at the journal bearing of Wollfarth [2]. In the secound part of the paper changes in the geometry of the end of the groove and a different shape of the feed hole run out are tested at the journal bearing of [2].

\section{Numerical Model}

\subsection{Geometrical Parameters}

The geometrical parameters of a journal bearing are given in this section. In figure 1 the different dimensions are illustrated. The inner cylinder with the radius $R_{1}$ is rotating with $\omega$ around the center of rotation. The fixed outer cylinder with the radius $R_{2}$ stands still.

The displacement of the inner cylinder is considered by the two eccentricities $e_{x}$ and $e_{y}$. Other important values can be calculate from the parameters given in figure 1 by the following equations 1 to 5 . 


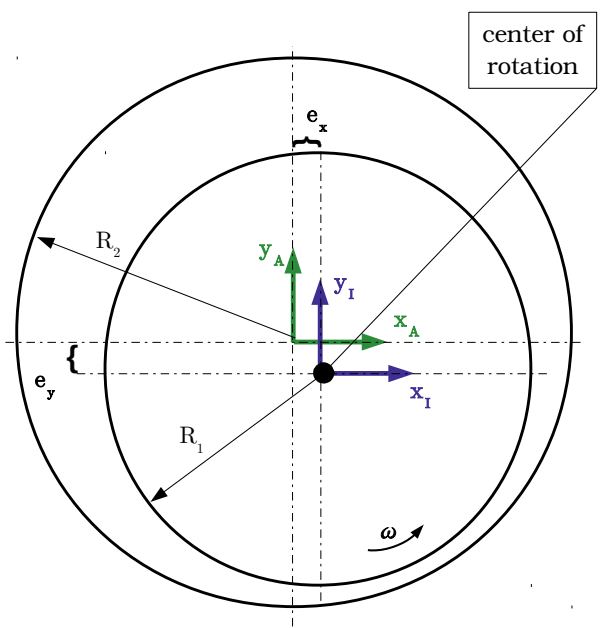

Fig. 1 schematic illustration of the principal journal bearing geometry

$$
\begin{gathered}
H_{0}=R_{2}-R_{1} \\
U_{1}=\omega \cdot R_{1} \\
R e=\frac{\Psi \cdot R_{1} \cdot U_{1}}{v} \\
\Psi=\frac{H_{0}}{R_{1}} \\
\varepsilon_{x, y}=\frac{e_{x, y}}{H_{0}}
\end{gathered}
$$

\subsection{Mathematical model}

The numerical solver is based on the finite-volume method. For a typical journal bearing of an internal combustion engine the Reynoldsnumbers is calculated $R e \approx 35$ [4]. That means that the flow is laminar. Furthermore the flow is isothermal since the bubble collaps is not considered. So the system of partial differential equations is reduced to the three dimensional conservation equations for mass (eq. 6) and momentum (eq. 7).

$$
\begin{gathered}
\frac{\partial \rho}{\partial t}+\nabla \cdot(\rho \mathbf{u})=0 \\
\frac{\partial \rho \mathbf{u}}{\partial t}+(\mathbf{u} \cdot \nabla) \mathbf{u}=\rho g-\nabla p+\mu \nabla^{2} \mathbf{u}
\end{gathered}
$$

To consider a secound phase and the phase change a transport equation have to be solved. This transport equation is given in eq. 8 .

$$
\frac{\partial \alpha}{\partial t}+\nabla \cdot(\alpha \mathbf{u})+\nabla \cdot\left[\alpha(1-\alpha) u_{\alpha}\right]=\frac{\dot{m^{+}+\dot{m^{-}}}}{\rho_{l}}
$$

The volume fraction $\alpha$ depends on the volume of liquid $V_{l}$ and the volume of vapour $V_{v}$ in a cell and is calculated with eq. 9.

$$
\alpha=\frac{V_{l}}{V_{l}+V_{v}}
$$

The third term in eq. 8 is only significant at the interface between the liquid and vapour phase. If $\alpha=1$ (liquid) or $\alpha=0$ (vapour) the term disappears. The velocity of the compression from the interface is given by the value $u_{\alpha}$. The source term on the right side of eq. 8 describes vaporisation $\dot{m}^{+}$and condensation $\dot{m}^{-}$. For this source term a bubble dynamic approach according to Sauer [5] is used. The transfered mass is calculated by eq. 10 .

$$
\begin{aligned}
\dot{m}= & \frac{\rho_{l} \cdot \rho_{v}}{\rho} \cdot \frac{3 \alpha_{N u c}}{R} \cdot\left(1-\alpha_{N u c}\right) \cdot \operatorname{sign}\left[p_{v}-p\right] \cdot \dot{R} \\
& \cdot\left\{\begin{array}{l}
C_{C}, \text { if } \operatorname{sign}\left[p_{v}-p\right]=-1 \\
C_{V}, \text { if } \operatorname{sign}\left[p_{v}-p\right]=1
\end{array}\right.
\end{aligned}
$$

The density $\rho$ and the viscosity $v$ are calculated as a mixture which depends on $\alpha$, see eq. 11 and eq. 12 .

$$
\begin{aligned}
& \rho=\rho_{l} \alpha+\rho_{v}(1-\alpha) \\
& v=v_{l} \alpha+v_{v}(1-\alpha)
\end{aligned}
$$

The ratio of vapour $\alpha_{N u c}$ depends on the radius of the nuclei $R$ and the number of nuclei $n_{0}$.

$$
\alpha_{N u c}=\frac{\frac{4}{3} \pi R^{3} n_{0}}{1+\frac{4}{3} \pi R^{3} n_{0}}
$$

For the bubble dynamic approach the velocity of the nuclei wall $\dot{R}$ is necessary. Therefore the simplified RayleighPlesset equation 14 is used.

$$
\dot{R}=\sqrt{\frac{2}{3} \cdot \frac{\left|p_{v}-p\right|}{\rho_{l}}}
$$

To adapt the solver for special cases two dimensionless constants $C_{C}$ and $C_{V}$ are used. They have a linear influence on the transfered mass of vapour or liquid. Close to the interface between vapour and liquid the eq. 7 is extended by the surface tension $\sigma$. The momentum equation in such regions is given in eq. 15 .

$$
\frac{\partial \rho \mathbf{u}}{\partial t}+(\mathbf{u} \cdot \nabla) \mathbf{u}=\rho g-\sigma \frac{1}{R} \mathbf{n}-\nabla p+\mu \nabla^{2} \mathbf{u}
$$

\subsection{Grid Generation}

For the grid generation the blockMesh tool from OpenFOAM is used. The important areas are the feedhole, the groove, the discharge hole and the gap between the inner and the outer cylinder. The feedhole and the discharge hole are meshed with a typical O-grid. For the groove and the gap a blockstructured mesh is used. To connect the different mesh shapes the option mergeMesh is used. A planar cut through the mesh is illustrate in figure 2. For the simulation of the experiment from Jakobsson et.al. [1] the grid is simplified. Both holes and the groove are not used so that only the gap has to be modelled. The hole mesh is used in the simulation of the experiment from Wollfarth [2]. 


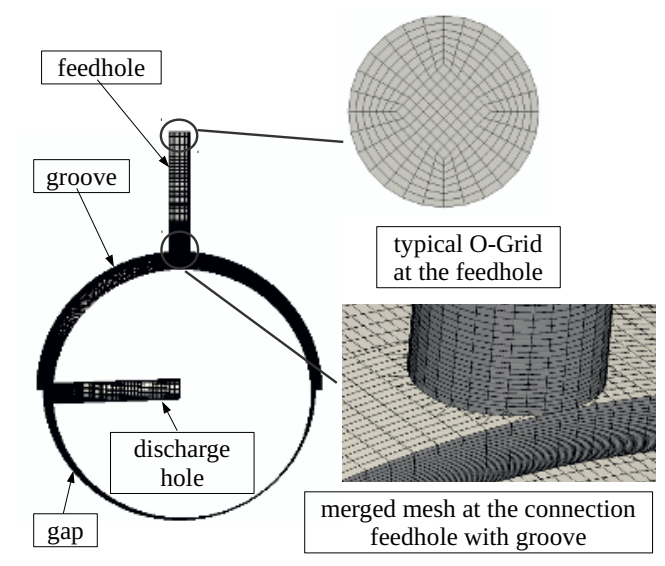

Fig. 2 numerical grid for the simulations

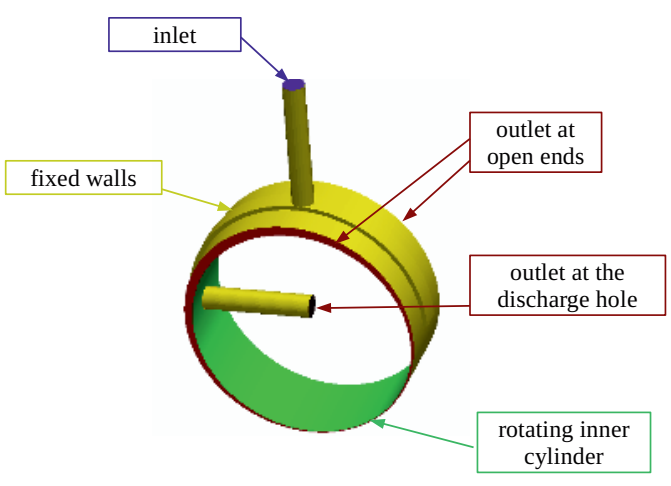

Fig. 3 boundaries at the hole numerical model

Table 1 boundary conditions for the simulation of the experiment from Jakobsson et.al. [1]

\begin{tabular}{llll}
\hline & vol. fraction & pressure & velocity \\
\hline inner cylinder & $\nabla \alpha=0$ & $\nabla p=0$ & $\omega=48,1 s^{-1}$ \\
outer cylinder & $\nabla \alpha=0$ & $\nabla p=0$ & $\mathbf{u}=\left(\begin{array}{lll}0 & 0\end{array}\right)^{T}$ \\
open ends & $\alpha=1$ & $p=1$ bar & $\nabla \cdot \mathbf{u}=0$ \\
\hline
\end{tabular}

\subsection{Boundary Conditions}

Cause of the two experiments, different boundaries are defined. For the simulation of the experiment from Jakobsson et.al. [1] only the fixed outer cylinder, the rotating inner cylinder and the outlet at the open ends has to be considered. At the simulation of the experiment from Wollfarth [2] furthermore an inlet at the feedhole, the outlet at the discharge hole, the fixed walls from feedhole, groove and discharge hole has to be considered. All these boundaries are shown in figure 3 . The boundary conditions for both experiments are listed in table 1 and 2

\subsection{Procedure}

In the real life the movement of the shaft in the bearing is very complex. It rotates around the centre axis, moves on a trajectory and is affected by the deformation of the housing. The consideration of all these effects leads to a complex CFD-Model resulting in long real time for the
Table 2 boundary conditions for the simulation of the experiment from Wollfarth [2]

\begin{tabular}{llll}
\hline & vol. fraction & pressure & velocity \\
\hline inlet & $\alpha=1$ & $p=15$ bar & $\mathbf{u}=f(p)$ \\
fixed walls & $\nabla \alpha=0$ & $\nabla p=0$ & $\mathbf{u}=(000)^{T}$ \\
inner cylinder & $\nabla \alpha=0$ & $\nabla p=0$ & $\omega=210 s^{-1}$ \\
outlets & $\alpha=1$ & $p=1$ bar & $\nabla \cdot \mathbf{u}=0$ \\
\hline
\end{tabular}

Table 3 identified values for different parameters

\begin{tabular}{ll}
\hline parameter & value $/$ range \\
\hline vapour pressure & $p_{v}=0,1 \mathrm{~Pa}$ \\
surface tension & $\sigma=0,0265 \mathrm{~N} / \mathrm{m}$ \\
liquid density & $\rho_{l}=800 \ldots 870 \mathrm{~kg} / \mathrm{m}^{3}$ \\
liquid viscosity & $v_{l}=1 \ldots 2 \cdot 10^{6} \mathrm{~m}^{2} / \mathrm{s}$ \\
vapour density & $\rho_{v}=9,8 \mathrm{~kg} / \mathrm{m}^{3}$ \\
vapour viscosity & $v_{v}=0,418 \cdot 10^{6} \mathrm{~m}^{2} / \mathrm{s}$ \\
\hline
\end{tabular}

simulation. A moderate time for the simulation is important for practical analysis. Therefore an alternative solution were found. In the first step the flow field is calculated with a steady-state solver with one fluid and without phase change. Here equation 6 and 7 are solved. The resulting values for pressure and velocity in every cell are the initial solution for the transient solution. The time for the simulation is the necessary time for turning the shaft about $2^{\circ}$. It is assumed that the position of the shaft $\left(e_{x}\right.$ and $\left.e_{y}\right)$ is known. For this short time the moving of the shaft on a trajectory and the deformation of the housing is neglected. The grid of the transient solution is the same like in the steady-state case. With this approach the special case of flow cavitation can be analised. This special type of cavitation in a journal bearing is founded by the deflection of the flow and a higher velocity caused by a smaller passage to pass by the flow. In this case the position of the discharge hole plays an important role and has to be considered.

\section{Adjustment of the Parameters}

\subsection{Selection of the Parameters}

In the equations 8 to 15 two constants and eight properties are included. For the liquid phase the values for density $\rho_{l}$ and viscosity $v_{l}$ are known. From the literature some values for the vapour pressure $p_{v}$ were found. A vapour pressure $p_{v} \approx 0,1 P a$ is in the most cases legal. It should be considered that oil consist of hydrocarbons. The different hydrocarbons steams at different pressures respectively temperatures. Mineral oil consists to $70 \%$ of paraffin hydrocarbons down to $\mathrm{C}_{18} \mathrm{H}_{38}$. For Eicosan $\left(\mathrm{C}_{20} \mathrm{H}_{42}\right)$ values for the density $\rho_{v}$ and viscosity $v_{v}$ of the vapour phase and the surface tension $\sigma$ are identified in $[6,7]$. For the number of nuclei, the start diameter of the nuclei and the constants $C_{C}$ and $C_{V}$ no values could be identified. This has to be done by different experimental settings according to the method Design of Experiments (DoE). Therefore the experiment from Jakobsson et.al. [1] is used. Here the length of the cavitation area at a constant width of the bearing is well-known with $l_{c a v}=96,9 \mathrm{~mm}$. 


\subsection{Design of Experiments}

To identify the relationship and the effect between different variables it is often necessary to perform a lot of experiments or simulations. Because of limited time and money the number of experiments or simulations are also limited. To get the most knowledge from the simulations the DoE is a good method. A model equation describes the relationship between the result and the variables. In the most cases a linear approach with interactions between the variables is sufficient to describe the relationship [8]. An example for a linear model with interactions is given in eq. 16 .

$$
\begin{aligned}
\phi & =a_{0}+\sum_{i=1}^{m} a_{i} \cdot \chi_{i}+\sum_{i=1}^{m} \sum_{j=1}^{i+1} a_{i j} \cdot \chi_{i} \cdot \chi_{j} \ldots \\
& +a_{k} \cdot \chi_{i} \cdot \chi_{j} \cdot \ldots \cdot \chi_{m}
\end{aligned}
$$

In the Jakobsson experiment the attribute $\phi$ is the cavitation length $l_{\text {cav }}$. The values $\chi_{i} \ldots \chi_{m}$ are functions from the unknown variables. This functions are unknown and the best way to identify these functions is to test different functions with considering the effect on the attribute $l_{c a v}$. The values $a_{0} \ldots a_{k}$ are the parameters of the model equation. These parameters should be identidied after the run of the experimental design. Equation 16 could also be written in matrix form. The parametervektor $\overline{\boldsymbol{a}}$ can than be determined by eq. 17.

$$
\overline{\boldsymbol{a}}=\left(\overline{\boldsymbol{F}}^{T} \cdot \overline{\boldsymbol{F}}\right)^{-1} \cdot \overline{\boldsymbol{F}}^{T} \cdot \overline{\boldsymbol{\phi}}
$$

At a two level design (every variable is tested at two different settings) the effect of the different variables can be calculated from the difference of the mean values at the high and low level. A large effect indicates that these variable has a large influence on the result. The effect can also be determined at interactions of variables.

\subsection{Testing the Approach}

The approach is verified with statistical tests. First the validity of the model equation is tested with regard to the experimental design. Then the determination of the regression is tested. For the validity of the model equation the simple coefficient of correlation $r_{o p}$ has to be determined, eq. 18.

$$
r_{o p}=\frac{s_{o p}}{s_{o} \cdot s_{p}}
$$

The covariance $s_{o p}$ obtains to the measured length of the simulation and the length calculated from the model equation.

$$
s_{o p}=\frac{1}{N-1} \sum_{i=1}^{N}\left(\phi_{i, \text { Meas }}-\bar{\phi}_{\text {Meas }}\right) \cdot\left(\phi_{i, \text { Mod }}-\bar{\phi}_{\text {Mod }}\right)
$$

The standard deviations $s_{o}$ and $s_{p}$ are also calculated from the length of the simulation and the calculated length.

$$
\begin{gathered}
s_{o}=\sqrt{\frac{1}{N-1} \sum_{i=1}^{N}\left(\phi_{i, \text { Meas }}-\bar{\phi}_{\text {Meas }}\right)^{2}} \\
s_{p}=\sqrt{\frac{1}{N-1} \sum_{i=1}^{N}\left(\phi_{i, \text { Mod }}-\bar{\phi}_{\text {Mod }}\right)^{2}}
\end{gathered}
$$

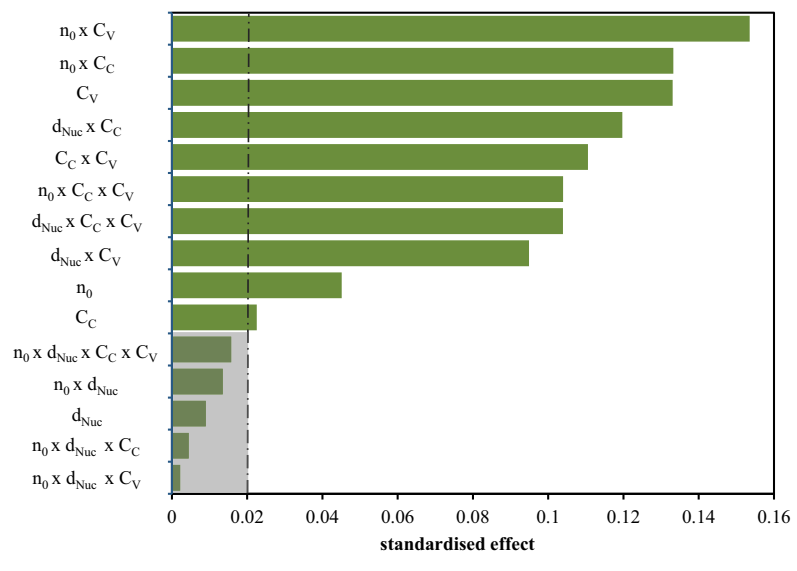

Fig. 4 standardised effect of every variable and interaction

With the simple coefficient of correlation and the number of simulations $N$ the following comparison has to be done:

$$
\frac{r_{o p}}{\sqrt{1-r_{o p}^{2}}} \cdot \sqrt{N-2}>t_{1-\lambda, f} .
$$

The value $t_{1-\lambda, f}$ is the percentile from the Student- distribution with the probability of error $\lambda$ and the degree of freedom $f=N-1$. A typical value for the probability of error is $\lambda=0,05$. If the comparison 22 is accepted the model equation is validated for the tested configuration of variables. The secound test is the determination of the regression. Therefore the multiple coefficient of correlation $r_{\chi_{i} \phi}$ has to be determined. For every parameter of the model equation a single multiple coefficient of correlation exists and is calculated with eq. 23 .

$$
r_{\chi_{i} \phi}=\sqrt{1-\frac{\operatorname{det}|\overline{\boldsymbol{S}}|}{s_{i}^{2} \cdot \operatorname{det}\left|\overline{\boldsymbol{S}}_{i i}\right|}}
$$

The value $\operatorname{det}|\overline{\boldsymbol{S}}|$ is the determinant of the covariance matrix, $s_{i}^{2}$ is the variance of the respective parameter and $\operatorname{det}\left|\overline{\boldsymbol{S}}_{i i}\right|$ is the subdeterminant of the covariance matrix. The subdeterminant is formed by removing from row $i$ and column $i$. With every multiple coefficient of correlation the following comparison has to be done:

$$
\frac{r_{\chi_{i} \phi}^{2}}{1-r_{\chi_{i} \phi}^{2}} \cdot \frac{N-k}{k-1}>F_{1-\lambda, f_{1}, f_{2}}
$$

Here $k$ stands for the number of parameters. The percentile of the Fisher-distribution $F_{1-\lambda, f_{1}, f_{2}}$ depends on the probability of error $\lambda$ and the degree of freedom $f_{1}=k-1$ and $f_{2}=N-k$. If the comparison 24 is accepted, the relationship between the variable and the model equation is confirmed.

\subsection{Analysis of the System}

In an older series of simulations the Jakobsson experiment were tested with a full factorial design on a coarse grid. From that series the effects of the different variables and interactions is determined and shown in figure 4. In figure 
Table 4 adjustment levels for the unknown variables

\begin{tabular}{lll}
\hline parameter & high level & low level \\
\hline number of nuclei & $n_{0, H}=10^{10}$ & $n_{0, L}=10^{8}$ \\
diameter of nuclei & $d_{N u c, H}=10 \mu \mathrm{m}$ & $d_{N u c, L}=2 \mu \mathrm{m}$ \\
constant for condensation & $C_{C, H}=1$ & $C_{C, L}=0,1$ \\
constant for vaporisation & $C_{V, H}=10$ & $C_{V, L}=1$ \\
\hline
\end{tabular}

4 it is shown that the diameter of the nuclei has an influence smaller than $2 \%$ and can be neglected. Also the interactions $n_{0} \cdot d_{N u c} \cdot C_{C} \cdot C_{V}, n_{0} \cdot d_{N u c} \cdot C_{C}, n_{0} \cdot d_{N u c} \cdot C_{V}$ and $n_{0} \cdot d_{N u c}$ have small influence on the result so they are also neglected. Every other variable and interaction is received, so eq. 25 is the linear model equation for the given case.

$$
\begin{aligned}
\phi_{\text {Modell }, 1} & =a_{0}+a_{1} \cdot \chi_{1}+a_{2} \cdot \chi_{3}+a_{3} \cdot \chi_{4}+\ldots \\
& \ldots+a_{4} \cdot \chi_{1} \chi_{3}+a_{5} \cdot \chi_{1} \chi_{4}+a_{6} \cdot \chi_{2} \chi_{3} \ldots \\
& \ldots+a_{7} \cdot \chi_{2} \chi_{4}+a_{8} \cdot \chi_{3} \chi_{4}+a_{9} \cdot \chi_{1} \chi_{3} \chi_{4} \ldots \\
& \ldots+a_{10} \cdot \chi_{2} \chi_{3} \chi_{4}
\end{aligned}
$$

The following relationship were tested and proved as the best:

$$
\begin{aligned}
& -\chi_{1}=\log _{10}\left(n_{0}\right), \\
& -\chi_{2}=\log _{10}\left(d_{N u c}\right), \\
& -\chi_{3}=\log _{10}\left(C_{C}\right), \\
& -\chi_{4}=\log _{10}\left(C_{V}\right) .
\end{aligned}
$$

In the case of four variables a full factorial design of the simulation runs is acceptable. That means for four variables on two adjustment levels, $2^{4}=16$ tests have to be performed. From older simulations some experience about the coarse range of the values is present. The high and low levels for every variable is given in table 4 .

With eq. 17 the parametervektor is determined. The statistical test 22 indicates with more than $95 \%$ probability that the model equation 25 is correct. Comparison 24 indicates for eight parameters also more than $95 \%$ probability of correctness. Only the parameters $a_{6}$ and $a_{7}$ have more than $90 \%$ probability of correctness, but this seems to be also ok. With model equation 25 a cavitation length $l_{\text {Cav }}=96,86 \mathrm{~mm}$ were determined with the following adjustment:

$$
\begin{aligned}
& -n_{0}=10^{10}, \\
& -n_{N u c}=2 \mu \mathrm{m}, \\
& -C_{C}=0,179, \\
& -C_{V}=5,58 .
\end{aligned}
$$

The cavitation length of the appendant simulation is $l_{\text {Cav }}=$ $109,4 \mathrm{~mm}$ and is to large. To improve the quality of the model equation first the full linear model equation with every interaction is used. This does not improve the result. Further steps with different model equations of secound order don"t show an advantage. To solve that problem a model equation with higher order terms is necessary. Therefore more simulations have to be done to train a neuronal network. The effort for that is very extensive and should not be carried out in the given case. Alternatively the simulation is chosen with the most closest cavitation length (the value is $l_{\text {Cav }}=97,2 \mathrm{~mm}$ ) as reference. The according settings are as follows:

$-n_{0}=10^{8}$,

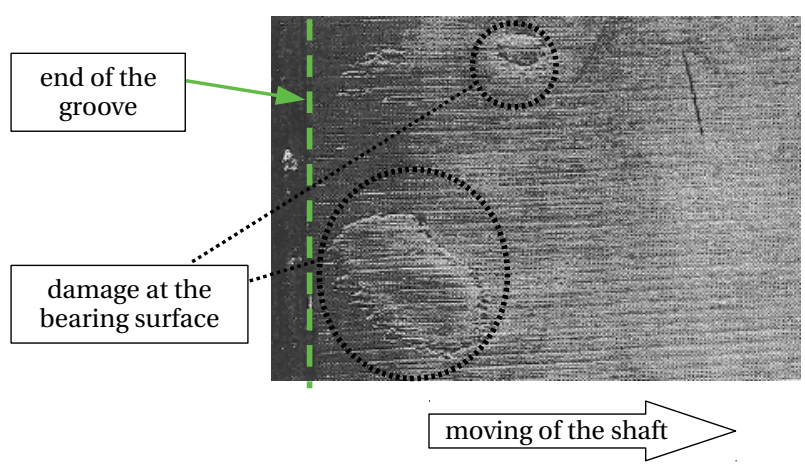

Fig. 5 damage of the bearing surface downstream the end of the groove

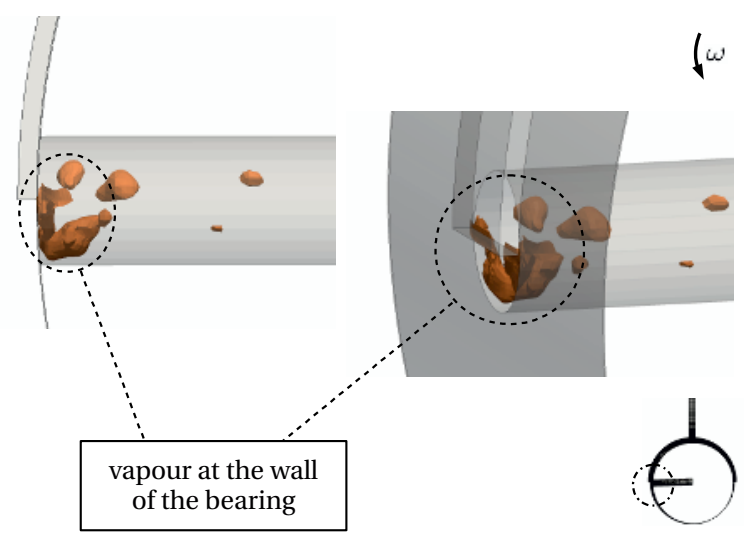

Fig. 6 vapour region downstream the end of the groove in the simulation

$$
\begin{aligned}
& -n_{N u c}=2 \mu m, \\
& -C_{C}=0,1, \\
& -C_{V}=1 .
\end{aligned}
$$

\section{Results}

\subsection{Validation of the Model}

In the first run the prepared model shall be validated with experimental data. Therefore the experiment from Wollfarth [2] is the reference. In this experiment the damage of the bearing surface is a indicator for the existence of cavitation respectively vapour at the bearing surface. In figure 5 the damage of the bearing surface is shown downstream the end of the groove. Therefore the discharge hole is placed at an angle $90^{\circ}$ downstream the feedhole. This is because the deflection of the flow is founded in the presence of the discharge hole at this region. In figure 6 the vapour region in the simulation is close to the surface of the bearing. This indicates the possibility of cavitation at the surface. The comparison of the cavitation regions in figure 5 with the vapour region in figure 6 shows a good agreement.

Another case of cavitation is shown in figure 7 . In this case the damage occurs downstream the feed hole at the surface of the bearing. The cavitation here is founded in the double deflection of the flow. The first deflection occurs at the intersection feedhole with groove and the secound at the intersection groove with discharge hole. For that case the discharge hole is placed at an angle $10^{\circ}$ downstream 
the feedhole. The related illustration from the simulation is shown in figure 8. Also in this case the vapour region is in good agreement with the damaged region at the bearing surface. The comparison between the numerical results and the damages from the experiment is very good. The conclusion is that the numerical solver gives a plausible solution for the flow cavitation in a journal bearing.

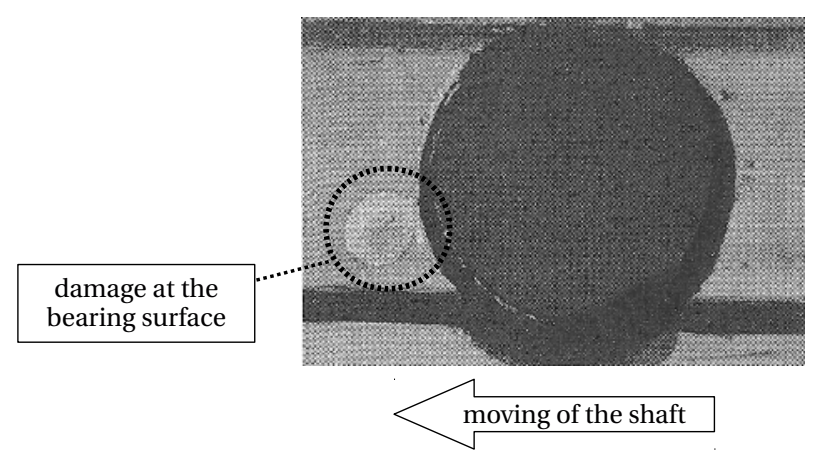

Fig. 7 damage of the bearing surface downstream the feedhole

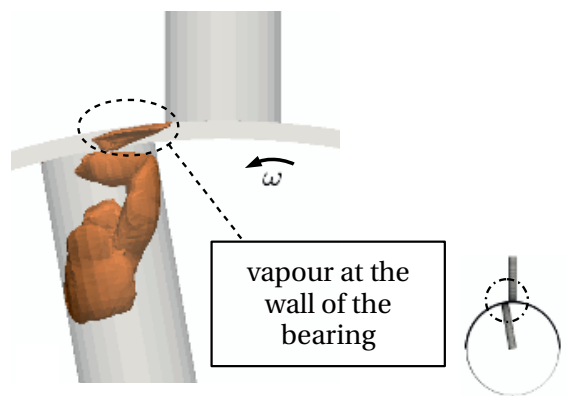

Fig. 8 vapour region downstream the feedhole in the simulation

\subsection{Investigation in Geometrical Changes}

After the validation of the model were done, the influence of different geometrical changes shall be tested. In the first series of simulations the effect of different shapes of the end of the groove is tested. A flow efficient shape should prevent the behaviour of cavitation. In Wollfarth [2] investigations on six different shapes were done. Three chamfer ends and three rounded ends were tested. The smoothest intersection should indicate the fewest affinity of cavitation. The vapour regions of the simulation is shown in figure 9 and figure 10. A qualitative analysis is shown in figure 11 where the volume of vapour of every shape is related to the unmachined shape. It is obvious that the amount of vapour decreases with smoother groove ends. In case of the smoothest intersection $\left(14^{\circ}\right.$ chamfer and rounded end with $R=20 \mathrm{~mm}$ ) the amount of vapour increases compared with the next smoothest intersection. The reason for that is founded in the high velocity in this region. The velocity of the original shape, the $14^{\circ}$ chamfer and the rounded end with $R=20 \mathrm{~mm}$ is shown in figure 12 . Caused by the smaller gap between the groove and the discharge hole the velocity increases and the pressure decreases. It is obvious that the main stream is more aligned to the wall and that the

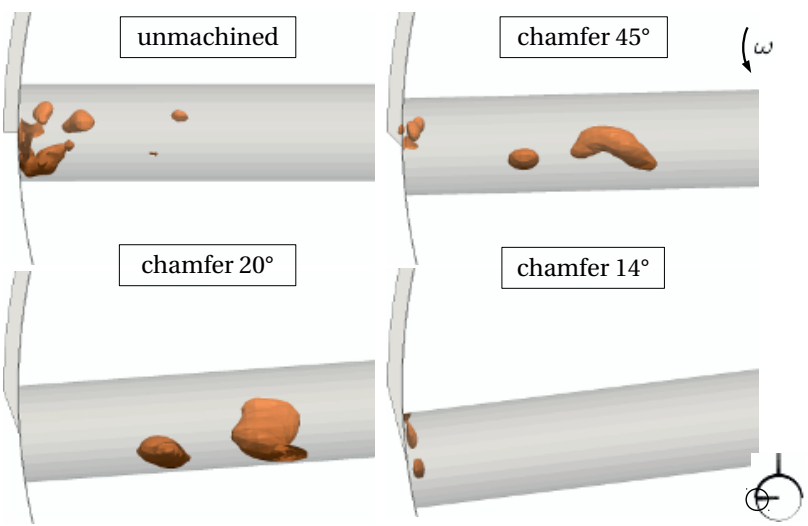

Fig. 9 three chamfer ends in comparison with the unmachined end of the groove

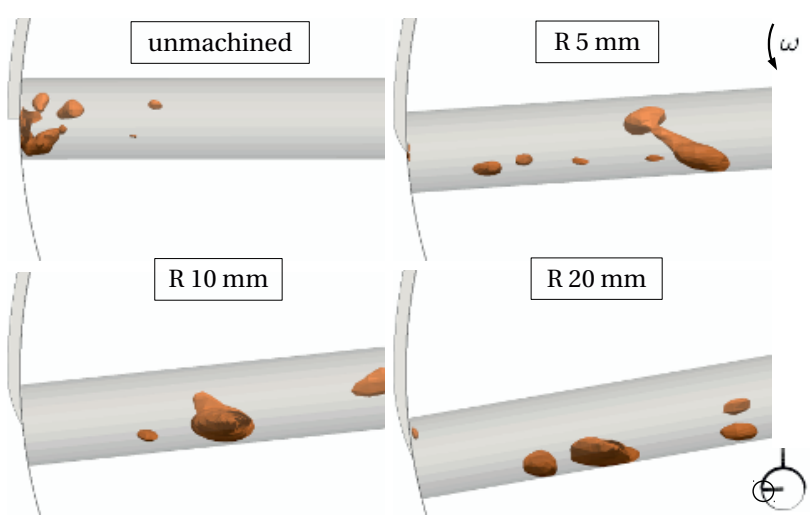

Fig. 10 three rounded ends in comparison with the unmachined end of the groove

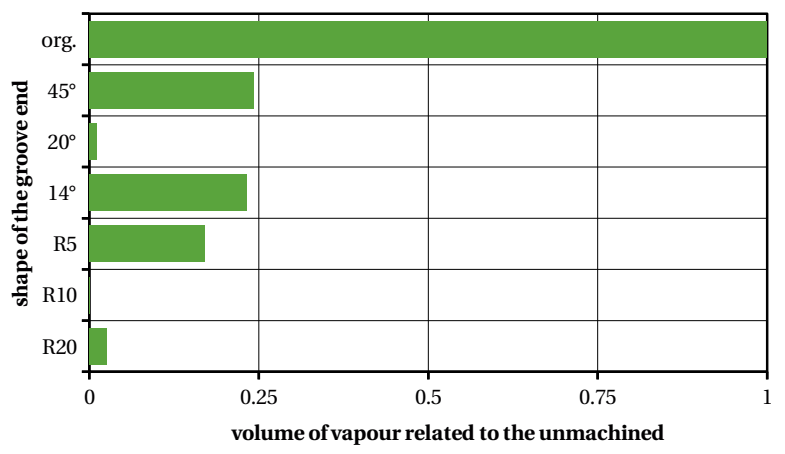

Fig. 11 relative volume of vapour related to the unmachined groove end

velocity is higher than in the original case. If the velocity is high enough the pressure underrun the vapour pressure and then the amount of vapour increases.

In another simulation the shape of the intersection from the feedhole to the groove is changed. A chamfer with (1x1) $\mathrm{mm}$ should improve the flow in that intersection so that the amount of vapour at the wall of the bearing increases. In figure 13 a comparison of the vapour region of the unmachined intersection with the chamfered intersection is illustrated. The amount of vapour at the wall of the bearing decreases. In the discharge hole the volume of vapour decreases. That is founded in the deflection of the flow at the intersection of the groove with the discharge hole. In that simulation the diameter of the discharge hole 


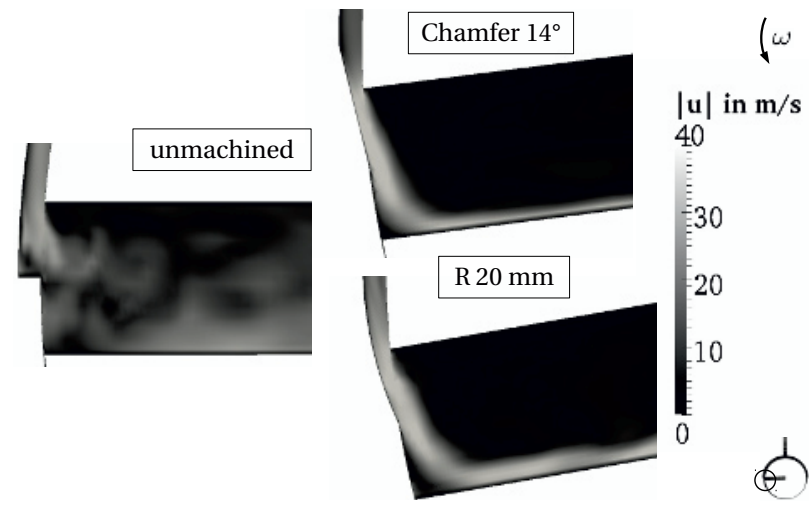

\section{Nomenclature}

Fig. 12 velocity in a planar cut of different shapes of the groove end

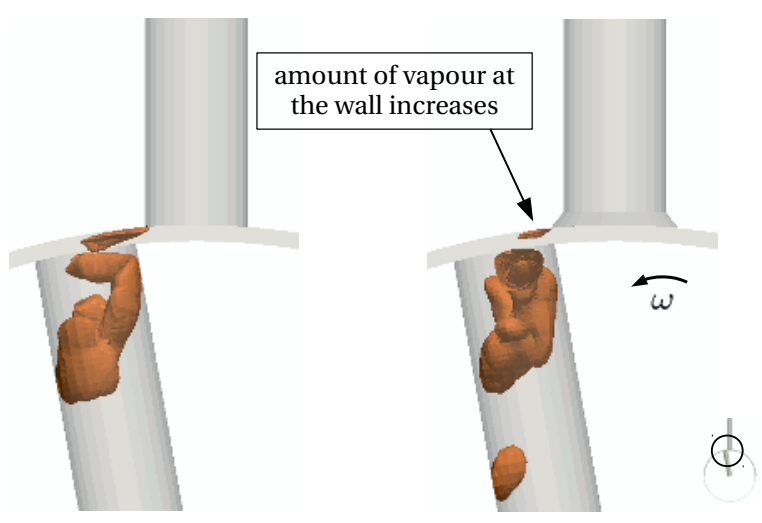

Fig. 13 velocity in a planar cut of different shapes of the groove end

is not equal to the width of the groove. In every other simulation the diameter of the feedhole and the discharge hole are equal to the width of the groove. To consider the chamfer a little modification at the model were done. This leads to the difference between the diameter and the width of the groove. However the simulation illustrates the decreasing risk of cavitation in the case of the chamfered intersection.

\section{Conclusion and Outlook}

In this work the possibility of simulating cavitating flow in a journal bearing geometry is shown. The constants and unknown properties for the specific numerical solver were found. With this settings the validation between the numerical results and the experimental data has been done. The results are satisfying. Furthermore the expected improvement of changes in geometrical details were shown in the simulation. This is a further reason to trust the results of the CFD simulation.

In further works the numerical gridgeneration can be improved. Details like chamfers, different shapes of the groove etc. should be optional adapted to the model. Otherwise other geometrical changes like different widths of the groove, heights of the groove and relative bearing clearances could be observed. 


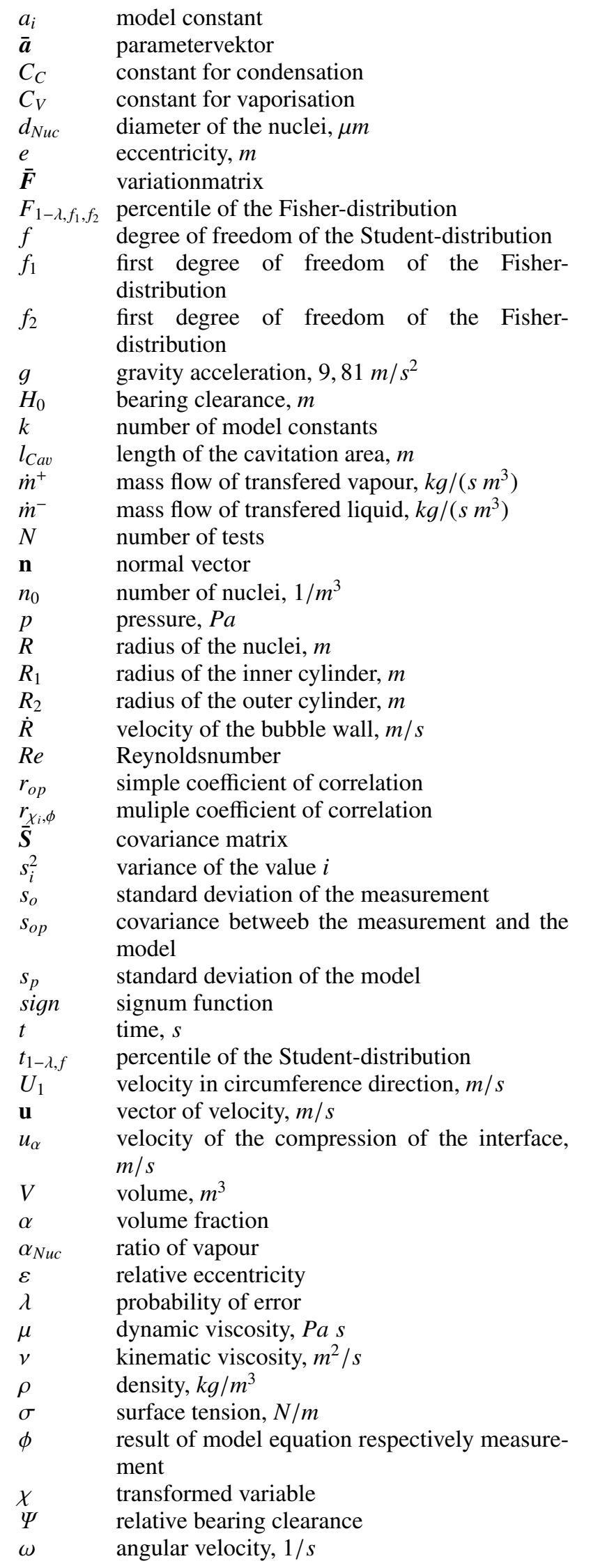

\section{Indices}

$1 \quad$ liquid

$\mathrm{v} \quad$ vapour

$\mathrm{x}, \mathrm{y}, \mathrm{z} \quad$ coordinates

\section{References}

1. B. Jakobsson, L. Floberg, Transactions of Chalmers University of Technology 190, (1957) pages 6-116

2. M. Wollfarth, Experimentelle Untersuchung der Kavitationserosion im Gleitlager ( $\mathrm{PhD}$ Thesis, University of Karlsruhe), (1995)

3. D.R. Garner, R.D. James, J.F. Warriner, Journal of Engineering for Power 102, (1980) pages 847-857

4. M. Riedel, Numerische Untersuchung von Kavitationseffekten in Radialgleitlagern (Master Thesis, University of Applied Science Zwickau), (2013)

5. J. Sauer, Instationär kavitierende Strömungen - Ein neues Modell basierend auf Front Capturing (VoF) und Blasendynamik (PhD Thesis, University of Karlsruhe), (2000)

6. Verein Deutscher Ingenieure (VDI); VDI-Wärmeatlas (Berlin), (2006)

7. Akron University, USA; ull.chemistry.uakron. edu/erd/Chemicals/4000/2639.html (2013)

8. J. Antony, Design of Experiments, for Engineers and Scientists (Oxford), (2003) 